\title{
Hepatotoxicity of Antimycotics Used for Invasive Fungal Infections: In Vitro Results
}

\author{
Sandra Doß, ${ }^{1,2}$ Heike Potschka, ${ }^{1}$ Fanny Doß, ${ }^{1}$ Steffen Mitzner, ${ }^{1,3}$ and Martin Sauer ${ }^{1,2}$ \\ ${ }^{1}$ Fraunhofer Institute for Cell Therapy and Immunology, EXIM, Rostock, Germany \\ ${ }^{2}$ Department of Anesthesiology and Intensive Care Medicine, University Hospital of Rostock, Schillingallee 35, Rostock, Germany \\ ${ }^{3}$ Department of Medicine, Division of Nephrology, University Hospital of Rostock, Schillingallee 35, Rostock, Germany \\ Correspondence should be addressed to Martin Sauer; martin.sauer@uni-rostock.de
}

Received 6 January 2017; Revised 17 February 2017; Accepted 21 March 2017; Published 4 April 2017

Academic Editor: Hartmut Jaeschke

Copyright (c) 2017 Sandra Doß et al. This is an open access article distributed under the Creative Commons Attribution License, which permits unrestricted use, distribution, and reproduction in any medium, provided the original work is properly cited.

Purpose. Drug-induced liver injury (DILI) is the most common cause of liver injury and a serious clinical problem; antimycotics are involved in approximately $3 \%$ of all DILI cases. The hepatotoxicity of many drugs, including the antimycotics, is poorly screened in human models. Methods. In a standardized assay the cytotoxicity on hepatocytes of different concentrations $(C \max , 5 \mathrm{x} C \mathrm{max}$, and 10x Cmax) of the antimycotics used for systemic infections was tested. Anidulafungin (ANI), liposomal amphotericerin B (L-AmB), caspofungin (CASPO), fluconazole (FLUCO), and voriconazole (VORI) were incubated with HepG2/C3A cells. After incubation, the viability of cells (XTT test, LDH release, trypan blue staining), the synthesis of albumin, the cytochrome 1A2 activity, and the cell death (DNA fragmentation) were determined. Kruskal-Wallis and Mann-Whitney tests were used for statistical analyses. Results. L-AmB, ANI, and CASPO showed a mild hepatotoxicity in the Cmax concentrations. Higher concentrations of anidulafungin led to a severe impairment of hepatocyte viability and function. The azoles FLUCO and VORI had a higher hepatotoxic potential in all concentrations. Conclusion. Antimycotics, especially azoles, used for systemic infections should be given with caution in patient with liver insufficiency or liver failure or high risk for this; therefore, therapeutic drug monitoring should be used. Further studies with this approach are encouraged.

\section{Introduction}

Drug-induced liver injury (DILI) is often responsible for hepatic dysfunction, the termination of drug development, or postmarket withdrawal of approved drugs [1-3]. DILI is a rare but serious clinical problem that is associated with significant morbidity and mortality. It is generally categorized as "idiosyncratic" or "intrinsic." These two forms of DILI are contrasted in their manifestation and diagnosis. Acetaminophen (APAP) is a common example for intrinsic hepatotoxins [4]; an APAP overdosage is the most frequent cause of drug-induced acute liver failure. It is dose-dependent and is reproducible in animal models [2]. Unfortunately, the underlying molecular mechanisms are not completely understood. Idiosyncratic DILI is typically rare and less reproducible. It occurs, when individuals are exposed to environmental or metabolic predispositions $[5,6]$.
More than 1000 drugs have been associated with DILI; DILI mimics severe liver injury and acute liver failure at therapeutic dose [7]. The early identification of hepatotoxic molecules and the prediction of DILI are main objectives of pharmacogenomics [3], but there is no "universal" in vitro screening approach. The current preclinical experimental models, including in vitro tests or the regulatory animal toxicity studies, might be insufficient for detecting certain types of human hepatotoxins [8]. These experimental models have strengths and weaknesses. After intensive and ongoing research, the prediction of human DILI using in vitro models remains relatively reliable. Animal models, however, are limited in detecting of human DILI. Data generated in animal models may not be translated into humans; moreover, these data may not be sufficient to predict DILI in humans, because there are species-specific differences in metabolism and pharmacokinetics $[9,10]$. In a retrospective study of 150 drugs, 
only $43 \%$ of human toxicities had been correctly predicted with animal tests compared to clinical experience [11].

Approximately $3 \%$ of DILI cases are caused by antimycotics (including acute liver failure) [12]. Invasive fungal infections increase and are associated with substantial morbidity and mortality rates, especially in immunosuppressed patients. Currently, there are only 14 invasive antimycotics available, which are poorly investigated for drug-induced hepatotoxicity $[13,14]$. Significant differences exist between the triazoles in regard to pharmacokinetics and pharmacodynamics, drug-drug interactions, concerning efficacy, and toxicity [15]. There is an increasing prevalence of resistant fungi $[16,17]$. For the effective personalized management of invasive fungal infections it is important to understand potential interactions and toxicities associated with antimycotics; moreover, it is important to understand the pharmacokinetics and pharmacodynamics of the antimycotics. Therefore, therapeutic drug monitoring (TDM), in combination with clinical assessment of the therapeutic effect, may help to optimize treatment results [18]. The aim of the presented study was to determine the hepatotoxicity of antimycotics used for systemic infections with human hepatocytes (HepG2/C3A) in an established in vitro cytotoxicity screening model [1922].

\section{Material and Methods}

2.1. Cell Cultures. HepG2/C3A (ATCC, ref. number CRL10741) were cultured in Dulbecco's modified Eagle's medium (DMEM, GIBCO Life Technologies, Darmstadt, Germany) supplemented with $10 \%$ fetal bovine serum (FBS, PAA Laboratories, Pasching, Germany), 1\% of $200 \mathrm{mM}$ L-glutamine (PAA), and $1 \%$ of antibiotics solution (Penicillin G: $10.000 \mathrm{IE} / \mathrm{ml} /$ Streptomycin: $10 \mathrm{mg} / \mathrm{ml}$; PAA). Cultures were maintained at $37^{\circ} \mathrm{C}$ in a $5 \% \mathrm{CO}_{2}$ humidified incubator. Cell concentration and vitality were assessed by trypan blue staining technique.

2.2. Drug Solutions. The lowest concentration of the different antifungals was the mean plasma level after induction of an i.v. therapy (Cmax) of anidulafungin (1x $100 \mathrm{mg}$ daily, ANI), caspofungin (1x $50 \mathrm{mg}$ daily, CASPO), fluconazole (2x $400 \mathrm{mg}$, FLUCO), voriconazole (1x $200 \mathrm{mg}$, VORI), and liposomal amphotericerin B (5 mg/kg/day i.v., L-AmB). Additionally to the Cmax, we tested two higher concentrations of the drugs $\left(5 \mathrm{x} C_{\max }, 10 \mathrm{x} C_{\max }\right):$ ANI $(7.5,37.5,75 \mu \mathrm{g} / \mathrm{ml}$; 2.25, 11.25, 22.5 mmol; Pfizer, NYC, USA) [23], CASPO (1, 5, $10 \mu \mathrm{g} / \mathrm{ml} ; 0.2,1.0,2.0 \mathrm{mmol}$; MSD, NJ, USA) [24], FLUCO $(2.5,12.5,25 \mu \mathrm{g} / \mathrm{ml} ; 4.5,22.5,45 \mathrm{mmol}$; ratiopharm, Ulm, Germany) [25], VORI (9, 45, $90 \mu \mathrm{g} / \mathrm{ml} ; 0.25,1.25,2.5 \mathrm{mmol}$; Pfizer, NYC, USA) [26], and L-AmB $(22.9,112.5,229 \mu \mathrm{g} / \mathrm{ml}$; 5.725, 28.625, 57.25 mmol; Gilead, CA, USA) [27].

2.3. Cytotoxicity on Hepatocytes Assay, pH Measurement. The antimycotics were tested in an established microtiter plate assay for screening of hepatotoxicity [19-22]. HepG2/C3A cells were seeded in 24 -well plates at 500.000 cells/well and were incubated for 72 hours with the different drugs in
$1 \mathrm{ml}$ medium or heparinized plasma from healthy volunteers (pooled plasma). Subsequently, fresh medium with the different concentrations of the antifungal were incubated with the cells for 72 hours again. Negative control served medium or plasma without agents and acetaminophen (APAP, 15,24 mM in medium, Sigma Aldrich, Seelze, Germany) was used as positive control. Each test batch was performed as a triplicate. The $\mathrm{pH}$ values (Radiometer, ABL, Willich, Germany) were screened before and after 72 hours and after 144 hours in the cell culture supernatant.

The following tests were carried out after 144 hours using the cells suspension or the cell culture supernatants.

Trypan blue exclusion assay $(0.4 \%$ (w/v); Sigma, Seelze, Germany) was used to determine the vitality and the number of cells with a C-Chip Neubauer improved haemocytometer (peqlab, Erlangen, Germany).

Albumin was determined nephelometrically from $0.2 \mathrm{ml}$ cell culture medium supernatant (Immage 800, Beckman Coulter GmbH, Germany) [28].

Plasma membrane integrity was determined by measuring lactate dehydrogenase (LDH) after 72 and 144 hours of incubation [29]. $0.2 \mathrm{ml}$ cell culture medium supernatant was photometrically measured by the change in the absorbance at $340 \mathrm{~nm}$ with the automated chemistry analyzer (Cobas Mira, Roche, Mannheim, Germany) according to the optimized standard method of the Deutsche Gesellschaft für Klinische Chemie (DGKC).

Cell viability was also determined using the 2,3-bis-(2methoxy-4-nitro-5-sulfophenyl)-2H-tetrazolium-5-carboxanilide inner salt (XTT, Roche Diagnostics GmbH, Mannheim, Germany), which is a colorimetric assay and is based on the ability of metabolic active cells to reduce the soluble tetrazolium salt (XTT) into an insoluble formazan with mitochondrial dehydrogenases [30,31]. At the start of the XTT-determination $2 \times 100 \mu \mathrm{l}$ cell suspension as duplicate was transferred to a transparent 96-well plate. After adding $100 \mu \mathrm{l}$ XTT-reaction reagent per well the increased formazan absorbance was read at a wavelength of $450 \mathrm{~nm}$ on a microplate reader (Anthos Reader 2001, Anthos Labtec Instruments, Austria) after one hour.

The activity of cytochrome P450 1A2 was determined by means of O-deethylization of 7- ethoxyresorufine to resorufin (EROD) [32] according to the method of Donato et al. [33]. Before measurement, hepatocytes stimulated with methylcholanthrene (3-MC, Sigma Aldrich, Seelze, Germany) for 72 hours were treated with $8 \mu \mathrm{M}$ ethoxyresorufin (Molecular Probes, Eugene, USA) and $10 \mu \mathrm{M}$ dicumarol (Sigma Aldrich, Seelze, Germany) for $1 \mathrm{~h}\left(37^{\circ} \mathrm{C}, 5 \% \mathrm{CO}_{2}\right)$. Resorufin concentration in the supernatants was measured at $530 \mathrm{~nm}$ (excitation) and $584 \mathrm{~nm}$ (emission) using a fluorescence multiwell plate reader (Fluoroskan Ascent Lab Systems, Vienna, USA). Concentrations were estimated against a resorufin standard curve (0 pmol, 10 pmol, 20 pmol, 40 pmol, and 80 pmol).

Fluorescence-activated cell sorting (FACS) analysis of DNA fragmentation was examined by the terminal deoxynucleotidyltransferase- (TdT-) mediated dUTP nick end labeling (TUNEL) method using the commercial APO-Direct Kit (BD Pharmingen, San Diego, CA, USA). Terminal deoxynucleotidyl transferase adds FITC-dUTP to each $3^{\prime}$-hydroxyl 
end of fragmented DNA, making it possible to measure the fragmentation of the DNA by the intensity of fluorescence.

The assay was performed following manufacturer's instructions. After treatment, cells were fixed with $1 \%$ paraformaldehyde in PBS $\left(4^{\circ} \mathrm{C}, 30 \mathrm{~min}\right)$, washed in $\mathrm{PBS}$, permeabilized with ice cold $70 \%$ ethanol, and stored at $-20^{\circ} \mathrm{C}$ overnight. The next day, after staining the cells the intensity of the fluorescence was determined by FACS analysis (FACS Calibur). In total, 5.000 events were collected per test sample. The results were analyzed by CellQuest software (both from BD Biosciences Systems, San Jose, CA, USA). The percentage of TUNEL negative and positive cells was presented.

2.4. Statistics. Statistical analysis was done with the Statistical Package for the Social Sciences (SPSS, Chicago, USA). Statistical differences between groups were analyzed using the Kruskal-Wallis test, because data were not normally distributed. Afterwards, the Mann-Whitney $U$ test for pairwise comparison was used. The results are expressed as median, 25 th and 75 th quartiles. A $p$ value of $<0.05$ was considered as significant.

\section{Results}

3.1. Effects of Antimycotics on Cell Proliferation and Vitality. The cell count was significantly decreased in the Cmax concentrations of fluconazole (FLUCO) and voriconazole (VORI) compared with the negative control in medium and plasma (Figure 1(a)). A further significant decrease in higher concentrations was only seen after incubation with anidulafungin (ANI) down to zero cells in the $10 \mathrm{x} C \max$ concentration (data not shown).

The vitality was decreased in the Cmax concentration after incubation with all antimycotics in medium and plasma (Figure 1(b)). Higher concentrations of ANI led to a markedly dose-dependent decrease of vitality (Figure 1(c)), which was not seen after incubations with the other antimycotics.

\subsection{Anidulafungin and Fluconazole Cause Loss of Cell Integrity} $(\mathrm{LDH})$. Significant increases of lactate dehydrogenase (LDH) in the Cmax concentrations were only seen after incubation with FLUCO compared with the negative control in medium and plasma (Figure 2(a)). After incubation with caspofungin (CASPO) significant lower values of LDH were measured. Dose-dependent effects were observed after incubation with ANI; Figure 2(b) shows the significant increase of LDH after incubation with ANI in higher concentrations (Figure 2(b)). At 3 days, the differences of $\mathrm{LDH}$ between the groups were greater than those at 6 days, but the same trends were seen (data not shown).

3.3. Effects of Antimycotic Drugs on the Activity of Mitochondrial Dehydrogenases in Hepatocytes. The activity of mitochondrial dehydrogenases was examined by the XTT test. Displayed in Figure 3(a), a significant increase was seen after incubation with ANI, FLUCO, and VORI in the Cmax concentrations in medium and plasma compared with the negative control. In contrast, incubation with ANI in higher concentrations (5x Cmax, 10x Cmax) led to significantly lower values in the XXT test (Figure 3(b)).

3.4. Liposomal Amphotericerin B Induced an Increase of CYP1A2 Activity. Liposomal amphotericerin B (L-AmB) and the positive control led to an increase of the cytochrome 1A2 activity; ANI, FLUCO, and VORI led to a significant decrease in the Cmax concentrations compared with the negative control in medium. Interestingly, incubation of the Cmax concentration of VORI with the test cells in plasma increased the cytochrome 1A2 activity (Figure 4). Dosedependent effects were not seen.

3.5. Effects of Antimycotics on Synthesis of Albumin. Significant increased values of albumin were detected in the cell culture supernatant after incubation with the Cmax concentrations of ANI, L-AmB, and FLUCO; in contrast, incubation with VORI in the Cmax concentration led to a significant decrease of the synthesis of albumin in medium and plasma (Figure 5). A dose-dependent decrease of albumin was observed after incubation with FLUCO and ANI; especially at higher concentrations of ANI (5x Cmax and 10x Cmax) synthesis of albumin decreased by $100 \%$ versus controls (data not shown).

3.6. Higher Concentrations of Anidulafungin Induced Cell Death (TUNEL Assay). The part of apoptotic or necrotic cells (DNA fragmentation) was low (5\%) after incubation with the Cmax concentrations of all used antimycotics and was comparable with the values of the negative control in medium and plasma (data not shown). A concentration-dependent influence on DNA fragmentation was only seen after incubation with ANI: at the $5 \mathrm{x}$ Cmax concentration in $32.8 \%$ of the hepatocytes apoptotic or necrotic cells determined and at the $10 \mathrm{x}$ Cmax concentration in $58.5 \%$ determined (Cmax concentration-ANI: $2.6 \%$ apoptotic cells).

\section{Discussions}

In our study we investigated the in vitro hepatotoxicity of antimycotics in clinical relevant concentrations (Cmax; $5 \mathrm{x}$ Cmax; 10x Cmax) [23-27] used for systemic infections with an established in vitro cytotoxicity screening model based on HepG2/C3A cells [19-22]. All drugs were tested in cell culture medium (with 10\% FBS) and plasma from healthy volunteers (pooled plasma) as a control. The idea behind this was to evaluate the influence of the different plasma protein bindings on the cytotoxicity of the used drugs. Only the CYP 1A2 activity in voriconazole-incubated cells was markedly higher in the control than in the medium. In all other results we did not see any difference between both approaches. We tested the viability of cells (XTT test, LDH release, and trypan blue staining), the synthesis of microalbumin, the cytochrome 1A2 activity, and the cell death (TUNEL assay). The use of XTT is an established test for the activity of mitochondrial dehydrogenases and the EROD-test for the activity of cytochrome 1A2, mainly involved in the metabolism of exogenous substances. In both tests, an increase as well as 


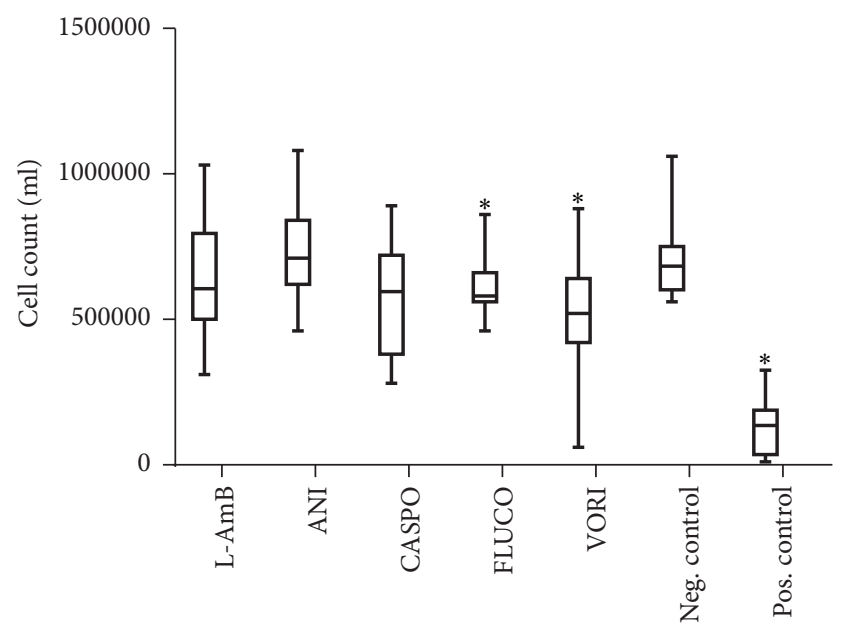

(a)

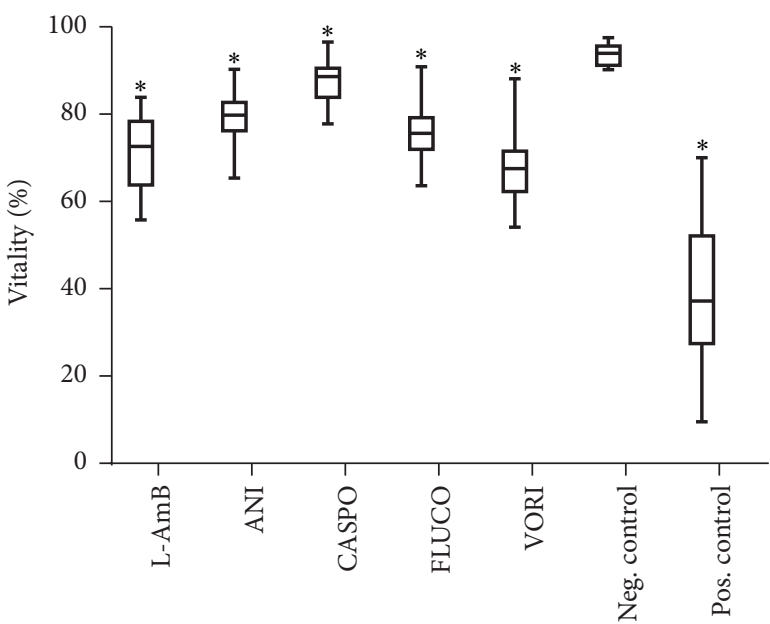

(b)

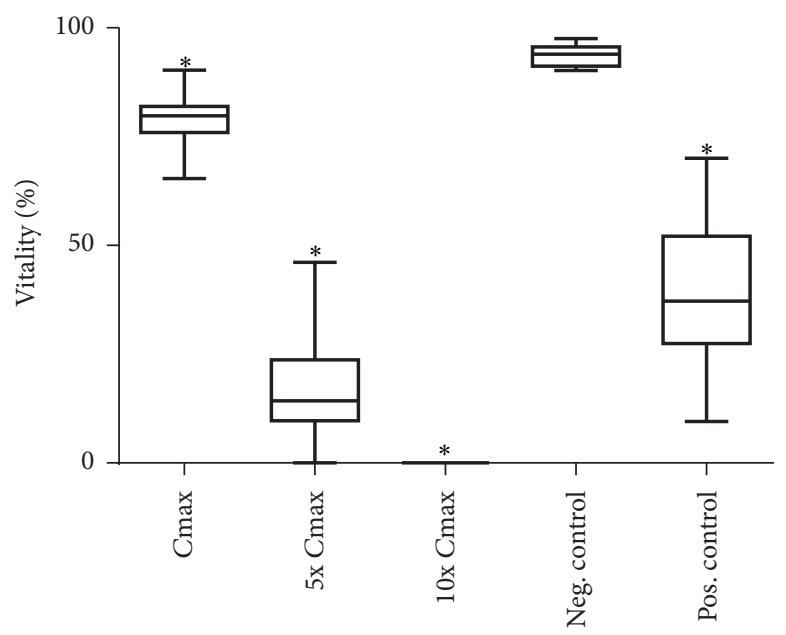

(c)

FIGURE 1: Effects of antimycotics agents on cell vitality and cell count in medium. Cells in culture were treated with $5.7 \mathrm{mM}$ liposomal amphotericerin B, $2.25 \mathrm{mM}$ anidulafungin, $0.2 \mathrm{mM}$ caspofungin, $4.5 \mathrm{mM}$ fluconazole, and $0.25 \mathrm{mM}$ voriconazole and untreated (negative control) and $15.24 \mathrm{mM}$ APAP (positive control) for 144 hours. Cells were counted in each treatment ((a), Cmax) and percent cell vitality was determined with trypan blue staining ((b), Cmax). Cell vitality at different doses of anidulafungin was further measured by trypan blue staining (c). Results are expressed as median and 25th/75th percentile, $n=20$ (biological and technical repeats). $*$ indicates significance of $p<0.05$ against the negative control.

a decrease of activity is a marker for toxic reactions of cells on different agents [30-33].

The human hepatocellular carcinoma cell line HepG2/ $\mathrm{C} 3 \mathrm{~A}$, a subclone of the hepatoma-derived HepG2 cell line, is an effective in vitro liver model that has been used to identify and evaluate compounds that may have hepatotoxic properties. The biosynthetic capabilities of C3A, similar to primary human hepatocytes, such as the production of many liverspecific plasma proteins [28], the presence of a functional cytochrome P450 toxin-processing enzyme system after stimulation [34, 35], and glucuronic- and sulfate-conjugation abilities were investigated and confirmed by independent investigators. Therefore, the cell line has the ability to carry out normal biotransformation reactions essential for the detoxification process. Flynn and Ferguson [36] and Liu et al. [37] treated HepG2/C3A cells with single, purified compounds and used a large number of biologically relevant assays to estimate the hepatotoxic potential of chemicals.

The C3A cell line does not completely mimic the behavior of liver lobule; nevertheless, C3A cells are a useful model for the predictive modeling of hepatotoxic agents [34], microfluidic devices [35], metabolome and tissue engineering approaches including 3D spheroid formation [38], and cell therapeutic applications, such as the extracorporeal liver assist device (ELAD) system [39].

The testing of anidulafungin, caspofungin, and fluconazole with C3A cells compared with primary isolated human hepatocytes provided similar results with the same trends focusing on cytochrome 1A2 activity, vitality, and activity of mitochondrial dehydrogenase (XTT or MTT test; own data, not shown). 


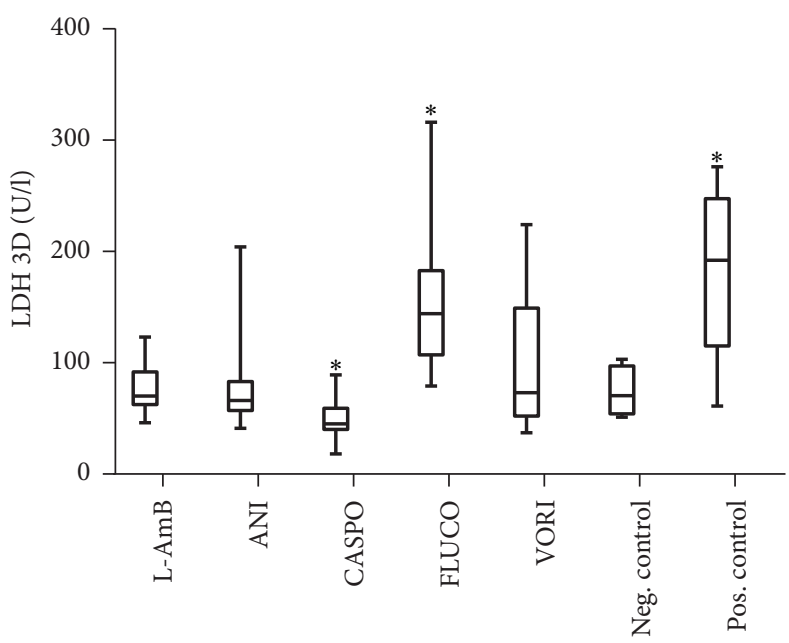

(a)

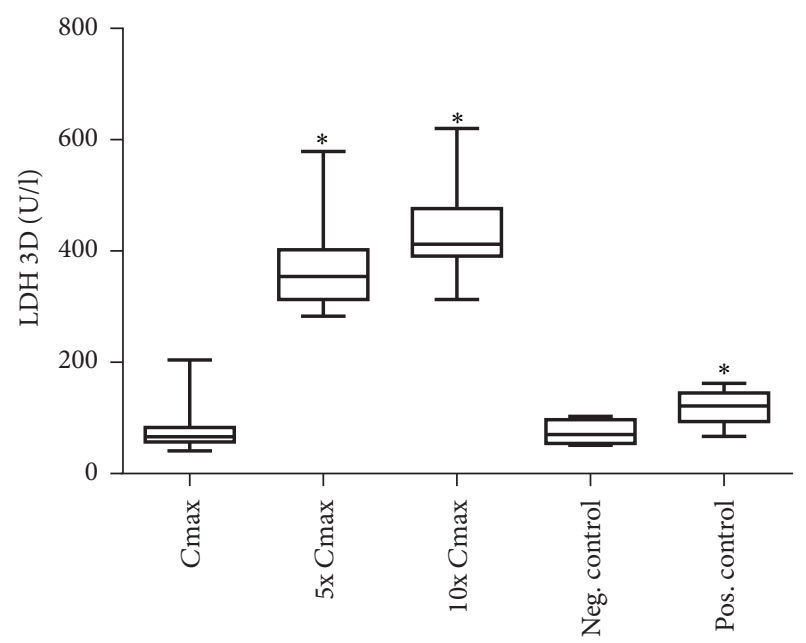

(b)

FIGURE 2: Effects of antimycotics agents on lactate dehydrogenase (LDH) release [U/l] in medium. Lactate dehydrogenase concentration in cultured C3A cells was measured after 72 hours of treatment with $5.7 \mathrm{mM}$ liposomal amphotericerin $\mathrm{B}, 2.25 \mathrm{mM}$ anidulafungin, $0.2 \mathrm{mM}$, caspofungin, $4.5 \mathrm{mM}$ fluconazole, $0.25 \mathrm{mM}$ voriconazole and untreated (negative control) and $15.25 \mathrm{mM}$ APAP (positive control) (a). LDH was further measured in different doses of anidulafungin (b). Results are expressed as median and 25th/75th percentile $(n=20$ biological and $n=40$ technical repeats). $*$ indicates significance of $p<0.05$ against the negative control.

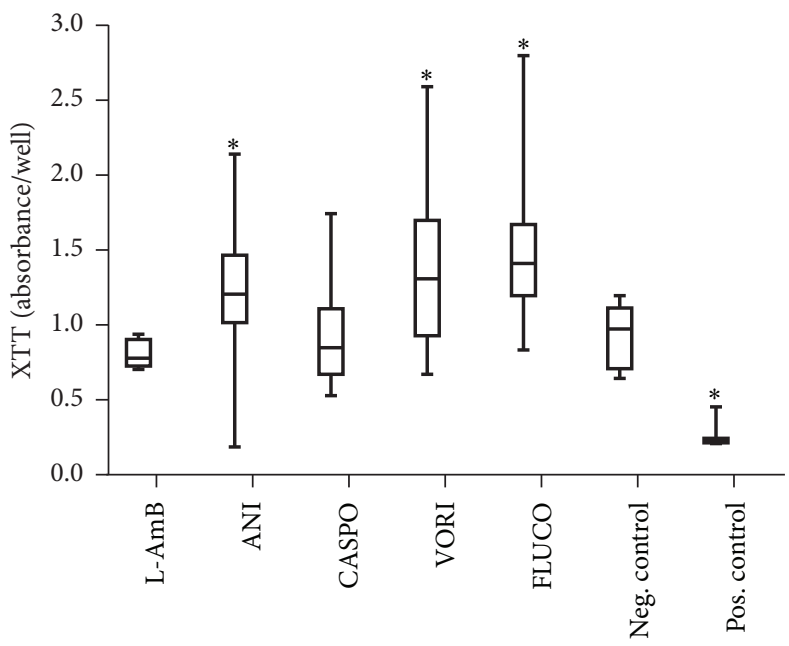

(a)

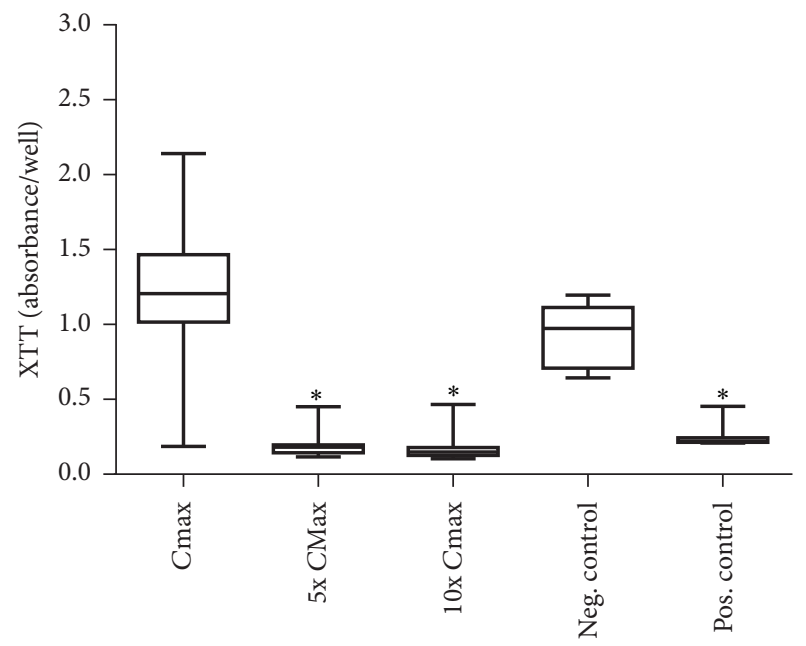

(b)

FIGURE 3: Effects of antimycotics agents on the activity of mitochondrial dehydrogenases: XTT test [absorbance/well] in medium. XTT assay of treated hepatocytes with $5.7 \mathrm{mM}$ liposomal amphotericerin $\mathrm{B}, 2.25 \mathrm{mM}$ anidulafungin, $0.2 \mathrm{mM}$ caspofungin, $4.5 \mathrm{mM}$ fluconazole, and $0.25 \mathrm{mM}$ voriconazole and untreated (negative control) and $15.25 \mathrm{mM}$ APAP (positive control). LDH concentrations in medium of antifungal treated hepatocytes after 144 hours (a) and dose-dependent effects of anidulafungin incubated hepatocytes (b). Results are expressed as median and 25 th/75th percentile ( $n=20$ biological and $n=40$ technical repeats). $*$ indicates significance of $p<0.05$ against the negative control.

Antifungal agents have been implicated in numerous cases of hepatotoxicity. Of all reported drug-related hepatotoxicity events, nearly $3 \%$ are mediated by antifungal agents [12]. On the other hand, the incidence of invasive fungal infections has increased steadily over the two decades [40]. The infections are characterized by high mortality and morbidity in immunocompromised patients. The most common species of human fungal pathogens are Aspergillus fumigatus
(50-90\%) and Candida albicans (20-40\%) [41]. Current treatment strategies for these infections have limitations in their spectrum of activity, drug interactions, pharmacokinetics, and pharmacodynamics properties, toxicity (dosing safety profile), and expense [42].

The risk of developing hepatotoxicity by antifungal agents is multifactorial; factors are preexisting liver disease, drugdrug interactions, the chemical properties of the antifungal 


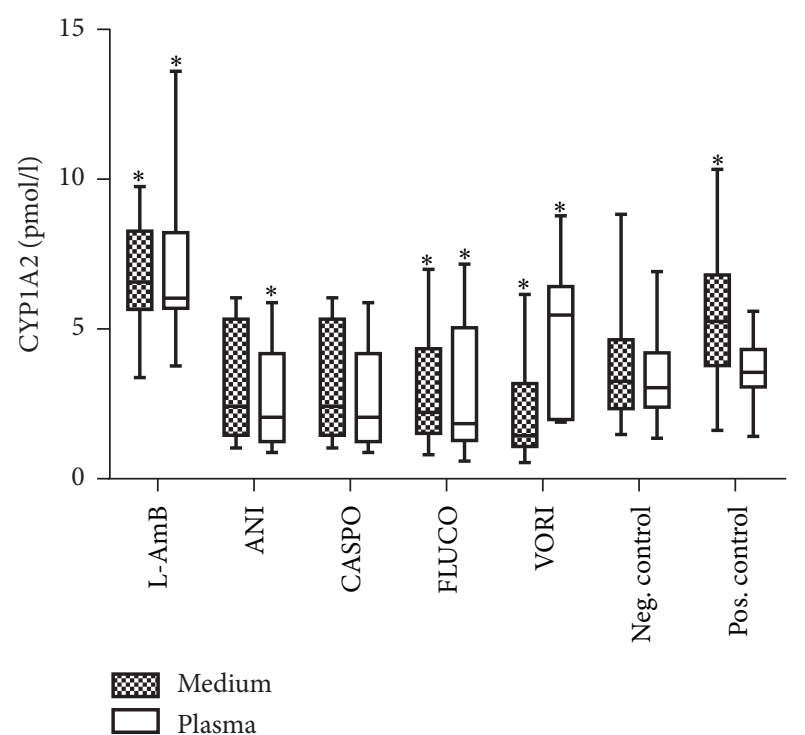

Figure 4: Effects of antimycotics agents on the cytochrome 1A2 activity (7-ethoxyresorufin O-deethylation and conversion of ethoxyresorufin to resorufin) [pmol/l] in medium and plasma. Cells in culture were treated with $5.7 \mathrm{mM}$ liposomal amphotericerin $\mathrm{B}$, $2.25 \mathrm{mM}$ anidulafungin, $0.2 \mathrm{mM}$ caspofungin, $4.5 \mathrm{mM}$ fluconazole, and $0.25 \mathrm{mM}$ voriconazole, and untreated (negative control) and 15.25 mM APAP (positive control). Results are expressed as median and 25th/75th percentile ( $n=20$ biological and $n=40$ technical repeats). * indicates significance of $p<0.05$ against the negative control.

agent, comorbidities, environmental factors (e.g., alcohol or the use of concomitant hepatotoxic drugs), genetic factors, the severity and treatment of the underlying disease, and liver involvement by the fungal infection $[12,43]$.

Liver injury caused by antifungal agents may vary from slight and asymptomatic abnormalities in liver enzymes to hepatotoxicity that occurs as potentially fatal fulminant liver failure [44]. Clinically significant liver injuries manifest mostly as acute hepatocellular, cholestatic, or mixed hepatocellular and cholestatic reactions [45]. The exact mechanisms of the toxicity of many of the antimycotics are unknown [46]; however, there has been no systematic evaluation of the liver toxicity associated with these treatments.

Liposomal amphotericin B (L-AmB) showed in our study with the C3A cell line a mild cytotoxicity on hepatocytes in all concentrations. A concentration-dependent manifestation of toxicity was not seen. The general toxicity of amphotericin $B$ is known; however, the lipid formulation demonstrated an improved safety profile compared to conventional amphotericin B. It is still unclear how frequently main side effects occur. Severe cases of L-AmB induced hepatotoxicity have been reported, but overall clinically apparent discontinuation of therapy and hepatotoxicity are rare [47]. Fischer et al. reported that $32 \%$ of hematopoietic stem cell transplantation (HSCT) patients, who received cumulative doses $>3.7 \mathrm{~g}$, developed a dose-dependent hepatotoxicity [47]. Wang et al. showed in a meta-analysis that the risk was only $0.7 \%$ for all lipid forms of amphotericerin B for a treatment

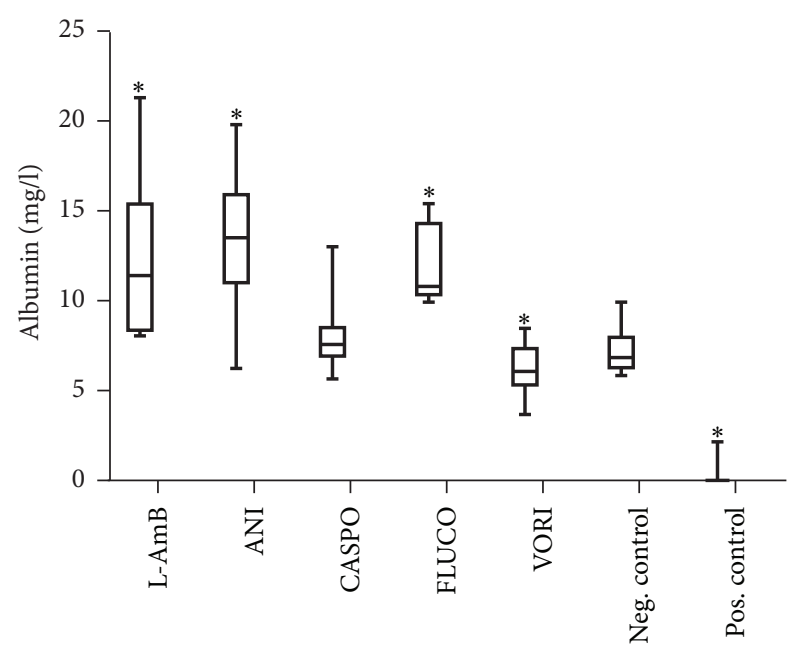

Figure 5: Release of albumin [mg/l] in medium. Treatments of cells with Cmax concentrations of $5.7 \mathrm{mM}$ liposomal amphotericerin $\mathrm{B}, 2.25 \mathrm{mM}$ anidulafungin, $0.2 \mathrm{mM}$ caspofungin, $4.5 \mathrm{mM}$ fluconazole, $0.25 \mathrm{mM}$ voriconazole, and untreated (negative control) and $15.25 \mathrm{mM}$ APAP (positive control). Results are expressed as median and 25 th/75th percentile, $n=20$ (biological and technical repeats). * indicates $p<0.05$ against the negative control.

discontinuation due to liver injury [44]. The L-AmB-induced hepatotoxicity in animal experiments is probably caused by the lipid component of the drug, but this has not yet been confirmed in humans (data in humans are lacking) [48].

The azoles fluconazole and voriconazole showed a higher toxic potential on hepatocytes in all concentrations in our study; especially in voriconazole-incubated cells these effects were more pronounced. Interestingly, these effects were not dose-dependent.

The incidence of mild transient liver damage associated with azole drugs is $2-12 \%$ [47]. A clear dose or time course relationship is not known for the azoles, but the use of voriconazole leads to liver damage. The toxicity is usually hepatocellular but can rarely occur as cholestatic or both $[49,50]$. Predominantly, the cholestatic or the mixed pattern of liver damage recurs with the restoration of fluconazole treatment [44, 51]. All azole antifungals are metabolized in the liver; while fluconazole is metabolized only minimally, itraconazole, voriconazole, and posaconazole are highly dependent on the metabolism for drug elimination [15]. All cases of hepatocellular-cholestatic liver injury were observed by fluconazoletreatments [52]. The mechanism of liver damage is unknown, but it is different from other triazole antifungal drugs, because fluconazole is excreted primarily in the urine and is the least potent as substrate or inhibitor of hepatic metabolism. Voriconazole-induced severe hepatotoxicity and treatment have been reported; however, hepatotoxicity was reversible, when ending therapy. Denning et al. reported in $15 \%$ of adult patients, receiving voriconazole, about abnormalities in liver enzymes with treatment discontinuation [53]. In patients, the observed pattern of liver damage was not uniform; it included a mixed hepatocellular-cholestatic, hepatocellular, or cholestatic injury [53]. For all patients a careful monitoring 
of liver enzymes is recommended receiving azole therapy. Abnormalities in liver enzymes, although reversible and rare after termination of treatment, are the most common laboratory abnormalities in voriconazole-treated patients [54]. Voriconazole appears to have a higher risk of liver damage than other modern antifungals $[55,56]$. In summary, voriconazole should be used with caution in patients with liver dysfunction or liver failure [54].

In this study, we observed a mild cytotoxicity on hepatocytes of echinocandin (anidulafungin, caspofungin) in the $C_{\max }$ concentration. In contrast, higher concentrations $(5 \mathrm{x}$ Cmax, 10x Cmax) of anidulafungin led to severe impairment of vitality, viability, and function of hepatocytes. In our study the lowest level of cytotoxicity on hepatocytes was found after incubation with caspofungin. Echinocandins have a low risk of hepatotoxicity and increased liver values; however, there are warnings in the prescribing information for all drugs of the group that hepatotoxicity may occur [57]. Clinically obvious hepatotoxicity appears only in isolated cases. Patients treated with the echinocandins observed transient increases in the liver enzymes that typically return to the baseline after the withdrawal of the therapy. Caspofungin can cause a higher rate of serum liver injury than micafungin or anidulafungin [58]. The cause of liver enzyme elevations during echinocandin therapy is partly unknown [44]. Echinocandins have been used safely in patients with preexisting hepatic impairment, namely, caspofungin in patients with chronic liver disease and postliver transplantation. Micafungin and anidulafungin have been used in patients with hepatic impairment; no worsening of the disease was observed [59, 60].

At the moment, it is still unclear if the hepatotoxic effect of the echinocandins may aggravate acute liver damage. Anidulafungin is not hepatically metabolized in contrast to caspofungin and micafungin [61]. All of them undergo a nonenzymatic chemical degradation to form a ring-opened product under neutral to basic conditions. The reactive intermediate formation is evident for the hepatotoxic potential; particularly anidulafungin generates a reactive intermediate able to trap glutathione [62-64].

Liver enzyme anomalies under caspofungin therapy are rare $[65,66]$. The coadministration of cyclosporine increased caspofungin exposure and has been associated with elevated liver transaminase levels in volunteers for a short time [67]. Compared to treatments with fluconazole and AmB, the incidence of liver damage in caspofungin-treated patients is lower. In a controlled study comparing caspofungin and $\mathrm{AmB}$ in 224 adults with invasive candidiasis, patients with caspofungin-treatment showed a lower ALT $(3.7 \%$ versus $8.1 \%)$, ALP (8.3\% versus $15.6 \%)$, and bilirubin (2.8\% versus $8.9 \%)$ serum levels abnormalities and no severe hepatic side effects [68]. In a study of 83 patients with invasive aspergillosis treated with caspofungin for over 20 days, ALT elevations occurred only in one patient and no serious drug-related hepatic side effects were reported [69]. Higher drug levels of caspofungin in patients with moderate liver insufficiency were found in nearly $75 \%$ of cases. A dose reduction to $35 \mathrm{mg}$ daily, after the $70 \mathrm{mg}$ administration dose, is recommended in the package insert; however, clinical experience with the use of caspofungin in patients with severe hepatic impairment (Child-Pugh score of $>9$ ) is very low [65].

The use of anidulafungin is rarely associated with adverse events and does not appear to be significantly hepatotoxic $[57,70]$. Saliba et al. found in a randomized, double blind study of anidulafungin versus fluconazole for the treatment of esophageal candidiasis an increase of liver enzymes including gamma glutamyl transpeptidase $(\gamma$-GT) $(1.3 \%$ in both groups) and AST ( $0.3 \%$ anidulafungin versus $2.3 \%$ of patients treated with fluconazole) [70]. In contrast to our results, according to the package insert no dosing adjustments of anidulafungin are necessary in patients with mild, moderate, or severe hepatic impairment [71].

\section{Conclusion}

In our in vitro liver model we assessed cytotoxicity of antimycotics. Concerning cytotoxic safety assessment, liposomal amphotericin B and caspofungin showed mild hepatotoxicity. The azole fluconazole and voriconazole showed a higher hepatotoxic potential; anidulafungin showed in higher concentrations ( $5 \mathrm{x} C \max , 10 \mathrm{x} C \max )$ a severe impairment of hepatocyte vitality, viability, and function. The mechanisms for the hepatotoxicity are unclear and need further investigations in suitable in vitro and in vivo models.

Azoles used for the therapy of systemic infections should be given with caution in patients with liver insufficiency and liver failure or high risk for this. Therefore, therapeutic drug monitoring (TDM), particularly during therapy with anidulafungin, may help to optimize the safety of therapy.

\section{Conflicts of Interest}

None of the authors have any conflicts of interest to declare.

\section{Authors' Contributions}

All authors were involved in the design, the performance, and analysis of the study.

\section{Acknowledgments}

This study relied solely on financial resources of the University of Rostock and the European Regional Development Fund (EFRE) and European Social Fund (ESF), Grant no. AU 09 046:ESF/IV-BM-B35-0005/12. The authors want to thank Dr. Stephanie Koch and Mr. Georg Richter for their very valuable technical support.

\section{References}

[1] P. B. Watkins, "Drug safety sciences and the bottleneck in drug development," Clinical Pharmacology and Therapeutics, vol. 89, no. 6, pp. 788-790, 2011.

[2] R. A. Roth and P. E. Ganey, "Intrinsic versus idiosyncratic druginduced hepatotoxicity-two villains or one?" Journal of Pharmacology and Experimental Therapeutics, vol. 332, no. 3, pp. 692-697, 2010. 
[3] S. Russmann, A. Jetter, and G. A. Kullak-Ublick, "Pharmacogenetics of drug-induced liver injury," Hepatology, vol. 52, no. 2, pp. 748-761, 2010.

[4] E. Björnsson, P. Jerlstad, A. Bergqvist, and R. Olsson, "Fulminant drug-induced hepatic failure leading to death or liver transplantation in Sweden," Scandinavian Journal of Gastroenterology, vol. 40, no. 9, pp. 1095-1101, 2005.

[5] N. Kaplowitz, "Idiosyncratic drug hepatotoxicity," Nature Reviews Drug Discovery, vol. 4, no. 6, pp. 489-499, 2005.

[6] K. Begriche, J. Massart, M.-A. Robin, A. Borgne-Sanchez, and B. Fromenty, "Drug-induced toxicity on mitochondria and lipid metabolism: mechanistic diversity and deleterious consequences for the liver," Journal of Hepatology, vol. 54, no. 4, pp. 773794, 2011.

[7] "Hepatotoxicity: the adverse effects of drugs and other chemicals on the liver," in Hepatotoxicity: The Adverse Effects of Drugs and Other Chemicals on the Liver, H. J. Zimmerman, Ed., Lippincott Williams \& Wilkins, Philadelphia, Pa, USA, 2nd edition, 1999.

[8] H. Olson, G. Betton, J. Stritar, and D. Robinson, "The predictivity of the toxicity of pharmaceuticals in humans from animal data-an interim assessment," Toxicology Letters, vol. 102-103, pp. 535-538, 1998.

[9] J. Bailey, M. Thew, and M. Balls, "An analysis of the use of animal models in predicting human toxicology and drug safety," Alternatives to Laboratory Animals, vol. 42, no. 3, pp. 181-199, 2014.

[10] J. Bailey, M. Thew, and M. Balls, "Predicting human drug toxicity and safety via animal tests: can any one species predict drug toxicity in any other, and do monkeys help?" Alternatives to Laboratory Animals, vol. 43, no. 6, pp. 393-403, 2015.

[11] F. D. Sistare and J. J. DeGeorge, "Preclinical predictors of clinical safety: opportunities for improvement," Clinical Pharmacology and Therapeutics, vol. 82, no. 2, pp. 210-214, 2007.

[12] E. Raschi, E. Poluzzi, A. Koci, P. Caraceni, and F. De Ponti, "Assessing liver injury associated with antimycotics: concise literature review and clues from data mining of the FAERS database," World Journal of Hepatology, vol. 6, no. 8, pp. 601612, 2014.

[13] Center for Drug Evaluation \& Research and US Food and Drug Adminstration, "New and generic drug approvals 1998-2004," Center for Drug Evaluation \& Research, http://www.fda.gov/.

[14] M. A. Donnelley, E. S. Zhu, and G. R. Thompson, "Isavuconazole in the treatment of invasive aspergillosis and mucormycosis infections," Infection and Drug Resistance, vol. 9, pp. 79-86, 2016.

[15] E. S. D. Ashley, R. Lewis, J. S. Lewis, C. Martin, and D. Andes, "Pharmacology of systemic antifungal agents," Clinical Infectious Diseases, vol. 43, no. 1, pp. S28-S39, 2006.

[16] N. P. Wiederhold and T. F. Patterson, "Emergence of azole resistance in aspergillus," Seminars in Respiratory and Critical Care Medicine, vol. 36, no. 5, pp. 673-680, 2015.

[17] S. Seyedmonsavi, "The role of azoles in the management of azole-resistant aspergillosis: from the bench to the bedside," Drug Resistance, vol. 17, no. 3, pp. 37-50, 2014.

[18] B. G. J. Dekkers, M. Bakker, K. C. M. van der Elst et al., "Therapeutic drug monitoring of posaconazole: an update," Current Fungal Infection Reports, vol. 10, no. 2, pp. 51-61, 2016.

[19] M. Sombetzki, N. Koslowski, S. Doss et al., "Biosensor for hepatocellular injury corresponds to experimental scoring of hepatosplenic schistosomiasis in mice," BioMed Research International, vol. 2016, Article ID 1567254, 7 pages, 2016.
[20] M. Sauer, J. Altrichter, C. Haubner et al., "Bioartificial therapy of sepsis: changes of norepinephrine-dosage in patients and influence on dynamic and cell based liver tests during extracorporeal treatments," BioMed Research International, vol. 2016, Article ID 7056492, 2016.

[21] M. Sauer, C. Haubner, T. Mencke et al., "Impaired cell functions of hepatocytes incubated with plasma of septic patients," Inflammation Research, vol. 61, no. 6, pp. 609-616, 2012.

[22] M. Sauer, "Use of human hepatocytes for determining liver function and liver regeneration," (DE/2006 102006005526), 2007.

[23] J. A. Dowell, M. Stogniew, D. Krause, T. Henkel, and I. E. Weston, "Assessment of the safety and pharmacokinetics of anidulafungin when administered with cyclosporine," Journal of Clinical Pharmacology, vol. 45, no. 2, pp. 227-233, 2005.

[24] G. Krishna, D. Vickery, L. Ma et al., "Lack of pharmacokinetic drug interaction between oral posaconazole and caspofungin or micafungin," Journal of Clinical Pharmacology, vol. 51, no. 1, pp. 84-92, 2011.

[25] M. Kami, Y. Sawada, S.-I. Mori et al., "Serum levels of fluconazole in patients after cytotoxic chemotherapy for hematological malignancy," American Journal of Hematology, vol. 66, no. 2, pp. 85-91, 2001.

[26] T. A. Driscoll, H. Frangoul, E. R. Nemecek et al., "Comparison of pharmacokinetics and safety of voriconazole intravenous-tooral switch in immunocompromised adolescents and healthy adults," Antimicrobial Agents and Chemotherapy, vol. 55, no. 12, pp. 5780-5789, 2011.

[27] S. Sundar and J. Chakravarty, "Liposomal amphotericin B and leishmaniasis: dose and response," Journal of Global Infectious Diseases, vol. 2, no. 2, pp. 159-166, 2010.

[28] N. Krasteva, T. Groth, F. Fey-Lamprecht, and G. Altankov, "The role of surface wettability on hepatocyte adhesive interactions and function," Journal of Biomaterials Science, Polymer Edition, vol. 12, no. 6, pp. 613-627, 2001.

[29] K. Lorentz, R. Klauke, and E. Schmidt, "Recommendation for the determination of the catalytic concentration of lactate dehydrogenase at 37 degrees C. Standardization Committee of the German Society for Clinical Chemistry, Enzyme Working Group of the German Society for Clinical Chemistry," European Journal of Clinical Chemistry and Clinical Biochemistry, vol. 31, no. 12, pp. 897-899, 1993.

[30] D. A. Scudiero, R. H. Shoemaker, K. D. Paull et al., "Evaluation of a soluble tetrazolium/formazan assay for cell growth and drug sensitivity in culture using human and other tumor cell lines," Cancer Research, vol. 48, no. 17, pp. 4827-4833, 1988.

[31] A. Nowrouzi, K. Meghrazi, T. Golmohammadi et al., "Cytotoxicity of subtoxic AgNP in human hepatoma cell line (HepG2) after long-term exposure," Iranian Biomedical Journal, vol. 14, no. 1-2, pp. 23-32, 2010.

[32] J. H. Kelly and N. L. Sussman, "A fluorescent cell-based assay for cytochrome P-450 isozyme 1A2 induction and inhibition," Journal of Biomolecular Screening, vol. 5, no. 4, pp. 249-253, 2000.

[33] M. T. Donato, M. J. Gomezlechon, and J. V. Castell, "A microassay for measuring cytochrome P450IAl and cytochrome P450IIB1 activities in intact human and rat hepatocytes cultured on 96-well plates," Analytical Biochemistry, vol. 213, no. 1, pp. 29-33, 1993.

[34] C. Rodríguez-Antona, M. T. Donato, A. Boobis et al., "Cytochrome $\mathrm{P} 450$ expression in human hepatocytes and hepatoma 
cell lines: molecular mechanisms that determine lower expression in cultured cells," Xenobiotica, vol. 32, no. 6, pp. 505-520, 2002.

[35] J. M. Prot, C. Aninat, L. Griscom et al., "Improvement of HepG2/C3a cell functions in a microfluidic biochip," Biotechnology and Bioengineering, vol. 108, no. 7, pp. 1704-1715, 2011.

[36] T. J. Flynn and M. S. Ferguson, "Multiendpoint mechanistic profiling of hepatotoxicants in HepG2/C3A human hepatoma cells and novel statistical approaches for development of a prediction model for acute hepatotoxicity," Toxicology in Vitro, vol. 22, no. 6, pp. 1618-1631, 2008.

[37] Y. Liu, T. J. Flynn, M. S. Ferguson, E. M. Hoagland, and L. L. Yu, "Effects of dietary phenolics and botanical extracts on hepatotoxicity-related endpoints in human and rat hepatoma cells and statistical models for prediction of hepatotoxicity," Food and Chemical Toxicology, vol. 49, no. 8, pp. 1820-1827, 2011.

[38] T. Elkayam, S. Amitay-Shaprut, M. Dvir-Ginzberg, T. Harel, and S. Cohen, "Enhancing the drug metabolism activities of C3A-a human hepatocyte cell line-by tissue engineering within alginate scaffolds," Tissue Engineering, vol. 12, no. 5, pp. 1357-1368, 2006.

[39] A. J. Ellis, R. D. Hughes, J. A. Wendon et al., "Pilot-controlled trial of the extracorporeal liver assist device in acute liver failure," Hepatology, vol. 24, no. 6, pp. 1446-1451, 1996.

[40] A. H. Groll, P. M. Shah, C. Mentzel, M. Schneider, G. JustNuebling, and K. Huebner, "Trends in the postmortem epidemiology of invasive fungal infections at a University Hospital," Journal of Infection, vol. 33, no. 1, pp. 23-32, 1996.

[41] B. J. Park, K. A. Wannemuehler, B. J. Marston, N. Govender, P. G. Pappas, and T. M. Chiller, "Estimation of the current global burden of cryptococcal meningitis among persons living with HIV/AIDS," AIDS, vol. 23, no. 4, pp. 525-530, 2009.

[42] R. E. Lewis, "Current concepts in antifungal pharmacology," Mayo Clinic Proceedings, vol. 86, no. 8, pp. 805-817, 2011.

[43] V. J. Navarro and J. R. Senior, "Drug-related hepatotoxicity," New England Journal of Medicine, vol. 354, no. 7, pp. 731-739, 2006.

[44] J.-L. Wang, C.-H. Chang, Y. Young-Xu, and K. A. Chan, "Systematic review and meta-analysis of the tolerability and hepatotoxicity of antifungals in empirical and definitive therapy for invasive fungal infection," Antimicrobial Agents and Chemotherapy, vol. 54, no. 6, pp. 2409-2419, 2010.

[45] M. S. Padda, M. Sanchez, A. J. Akhtar, and J. L. Boyer, "Druginduced cholestasis," Hepatology, vol. 53, no. 4, pp. 1377-1387, 2011.

[46] F. P. Tverdek, D. Kofteridis, and D. P. Kontoyiannis, "Antifungal agents and liver toxicity: a complex interaction," Expert Review of Anti-Infective Therapy, vol. 14, no. 8, pp. 765-776, 2016.

[47] M. A. Fischer, W. C. Winkelmayer, R. H. Rubin, and J. Avorn, "The hepatotoxicity of antifungal medications in bone marrow transplant recipients," Clinical Infectious Diseases, vol. 41, no. 3 , pp. 301-307, 2005.

[48] G. W. Boswell, I. Bekersky, D. Buell, R. Hiles, and T. J. Walsh, "Toxicological profile and pharmacokinetics of a unilamellar liposomal vesicle formulation of amphotericin B in rats," Antimicrobial Agents and Chemotherapy, vol. 42, no. 2, pp. 263268, 1998.

[49] I. Amigues, N. Cohen, D. Chung et al., "Hepatic safety of voriconazole after allogeneic hematopoietic stem cell transplantation," Biology of Blood and Marrow Transplantation, vol. 16, no. 1, pp. 46-52, 2010.
[50] J. C. Song and S. Deresinki, "Hepatotoxicity of antifungal agents," Current Opinion in Investigational Drugs, vol. 6, no. 2, pp. 170-177, 2005.

[51] M. O. Gearhart, "Worsening of liver function with fluconazole and review of azole antifungal hepatotoxicity," Annals of Pharmacotherapy, vol. 28, no. 10, pp. 1177-1181, 1994.

[52] R. K. Scher, "Onychomycosis: therapeutic update," Journal of the American Academy of Dermatology, vol. 40, no. 6, part 2, pp. S21-S26, 1999.

[53] D. W. Denning, P. Ribaud, N. Milpied et al., "Efficacy and safety of voriconazole in the treatment of acute invasive aspergillosis," Clinical Infectious Diseases, vol. 34, no. 5, pp. 563-571, 2002.

[54] P. Solís-Muñoz, J. C. López, W. Bernal et al., "Voriconazole hepatotoxicity in severe liver dysfunction," Journal of Infection, vol. 66, no. 1, pp. 80-86, 2013.

[55] H. Foo and T. Gottlieb, "Lack of cross-hepatotoxicity between voriconazole and posaconazole," Clinical Infectious Diseases, vol. 45, no. 6, pp. 803-805, 2007.

[56] W. J. Heinz, G. Egerer, H. Lellek, A. Boehme, and J. Greiner, "Posaconazole after previous antifungal therapy with voriconazole for therapy of invasive aspergillus disease, a retrospective analysis," Mycoses, vol. 56, no. 3, pp. 304-310, 2013.

[57] A. Desai, A.-H. Schmitt-Hoffmann, S. Mujais, and R. Townsend, "Population pharmacokinetics of isavuconazole in subjects with mild or moderate hepatic impairment," Antimicrobial Agents and Chemotherapy, vol. 60, no. 5, pp. 3025-3031, 2016.

[58] D. P. Kontoyiannis, "Invasive mycoses: strategies for effective management," American Journal of Medicine, vol. 125, no. 1, pp. S25-S38, 2012.

[59] C. Doria, A. S. Bodzin, S. Vaccino, C. Daskalakis, S. Krawitz, and C. B. Ramirez, "A retrospective analysis of the use of caspofungin in recipients of liver transplant with a modified high index of suspicion for fungal infection. A critical review of mortality, acute cellular rejection, infections, and changes in the liver function tests while on caspofungin," Clinical Transplantation, vol. 25, no. 4, pp. 569-575, 2011.

[60] G. Auzinger, "Invasive candidiasis following liver transplantation and surgical complications," Mycoses, vol. 54, supplement 4, pp. 4-7, 2011.

[61] D. S. Jung, F. P. Tverdek, and D. P. Kontoyiannis, "Switching from posaconazole suspension to tablets increases serum drug levels in leukemia patients without clinically relevant hepatotoxicity," Antimicrobial Agents and Chemotherapy, vol. 58, no. 11, pp. 6993-6995, 2014.

[62] V. Ong, G. Hough, M. Schlosser et al., "Preclinical evaluation of the stability, safety, and efficacy of CD101, a novel echinocandin," Antimicrobial Agents and Chemotherapy, vol. 60, no. 11, pp. 6872-6879, 2016.

[63] T. A. Baillie and A. E. Rettie, "Role of biotransformation in drug-induced toxicity: influence of intra- and inter-species differences in drug metabolism," Drug Metabolism and Pharmacokinetics, vol. 26, no. 1, pp. 15-29, 2011.

[64] L. Leung, A. S. Kalgutkar, and R. S. Obach, "Metabolic activation in drug-induced liver injury," Drug Metabolism Reviews, vol. 44, no. 1, pp. 18-33, 2012.

[65] J. Maertens, I. Raad, G. Petrikkos et al., "Efficacy and safety of caspofungin for treatment of invasive aspergillosis in patients refractory to or intolerant of conventional antifungal therapy," Clinical Infectious Diseases, vol. 39, no. 11, pp. 1563-1571, 2004.

[66] S. C. Deresinski and D. A. Stevens, "Caspofungin," Clinical Infectious Diseases, vol. 36, no. 11, pp. 1445-1457, 2003. 
[67] G. C. Mistry, E. Migoya, P. J. Deutsch et al., "Single- and multiple-dose administration of caspofungin in patients with hepatic insufficiency: implications for safety and dosing recommendations," Journal of Clinical Pharmacology, vol. 47, no. 8, pp. 951961, 2007.

[68] F. Saner, J. Gensicke, P. Rath et al., "Safety profile of concomitant use of caspofungin and cyclosporine or tacrolimus in liver transplant patients," Infection, vol. 34, no. 6, pp. 328-332, 2006.

[69] N. A. Kartsonis, J. Nielsen, and C. M. Douglas, "Caspofungin: the first in a new class of antifungal agents," Drug Resistance Updates, vol. 6, no. 4, pp. 197-218, 2003.

[70] F. Saliba, A. Pascher, O. Cointault et al., "Randomized trial of micafungin for the prevention of invasive fungal infection in high-risk liver transplant recipients," Clinical Infectious Diseases, vol. 60, no. 7, pp. 997-1006, 2015.

[71] J. A. Vazquez and J. D. Sobel, "Anidulafungin: a novel echinocandin," Clinical Infectious Diseases, vol. 43, no. 2, pp. 215-222, 2006. 


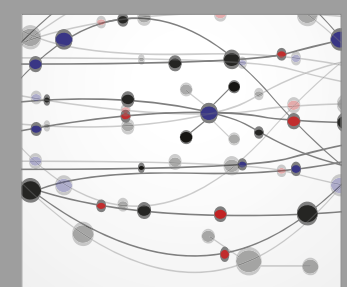

The Scientific World Journal
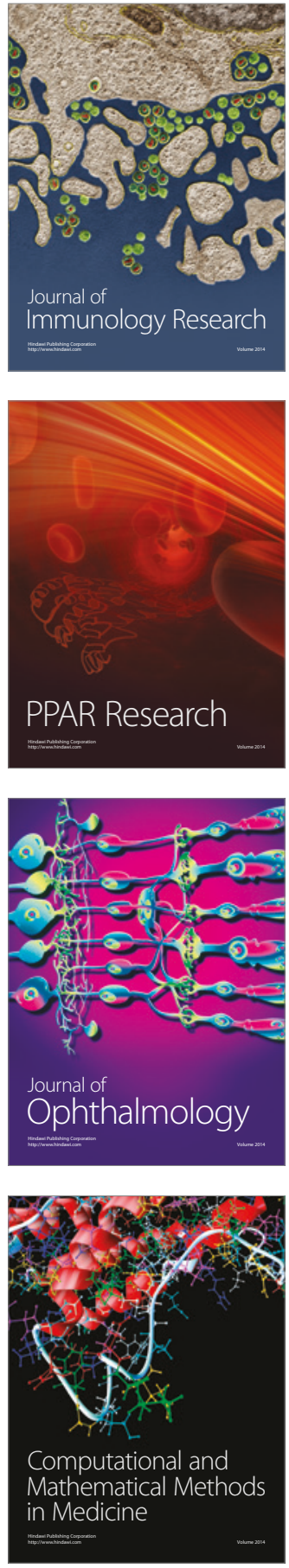

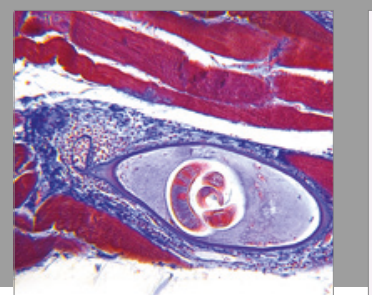

Gastroenterology Research and Practice
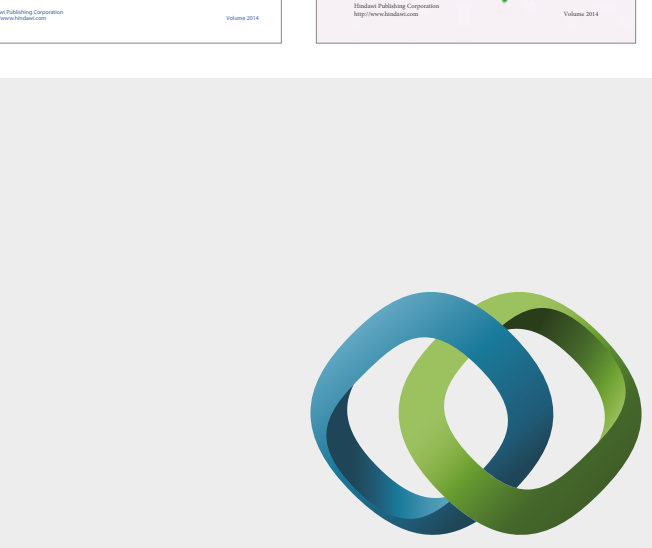

\section{Hindawi}

Submit your manuscripts at

https://www.hindawi.com
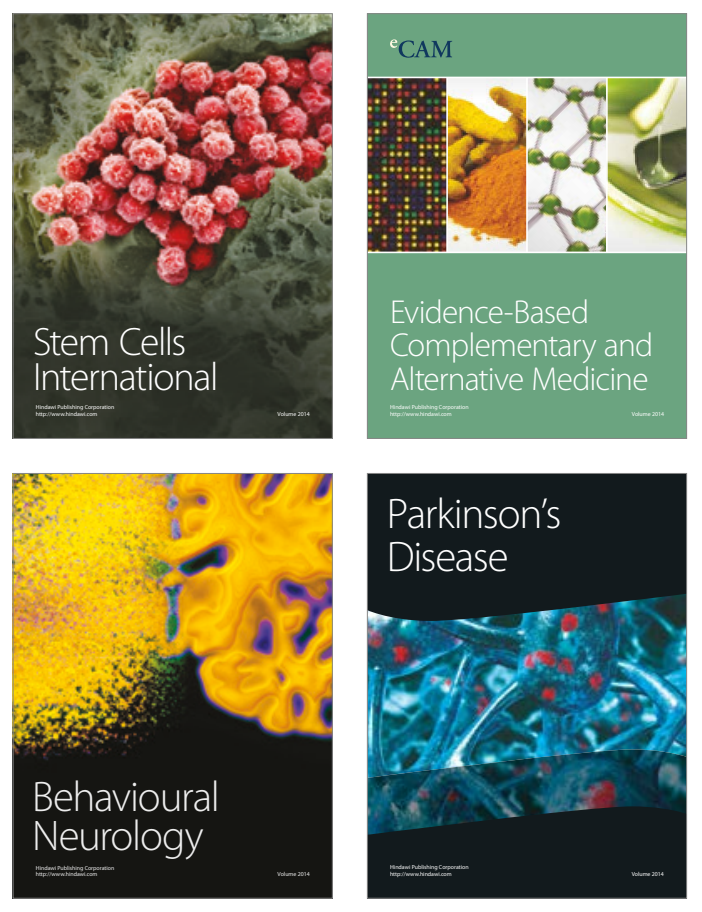
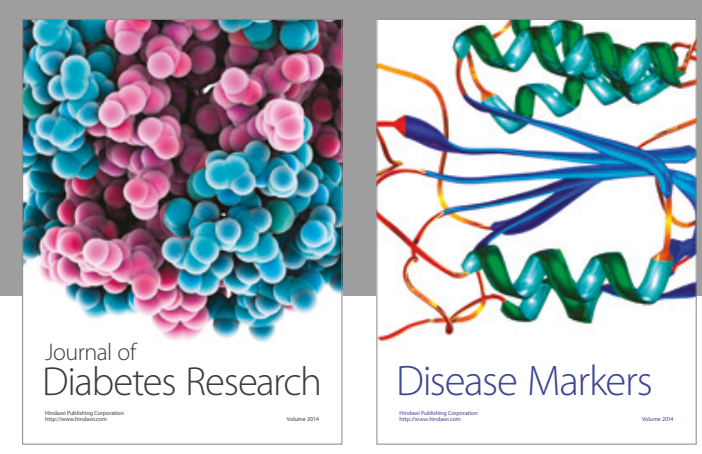

Disease Markers
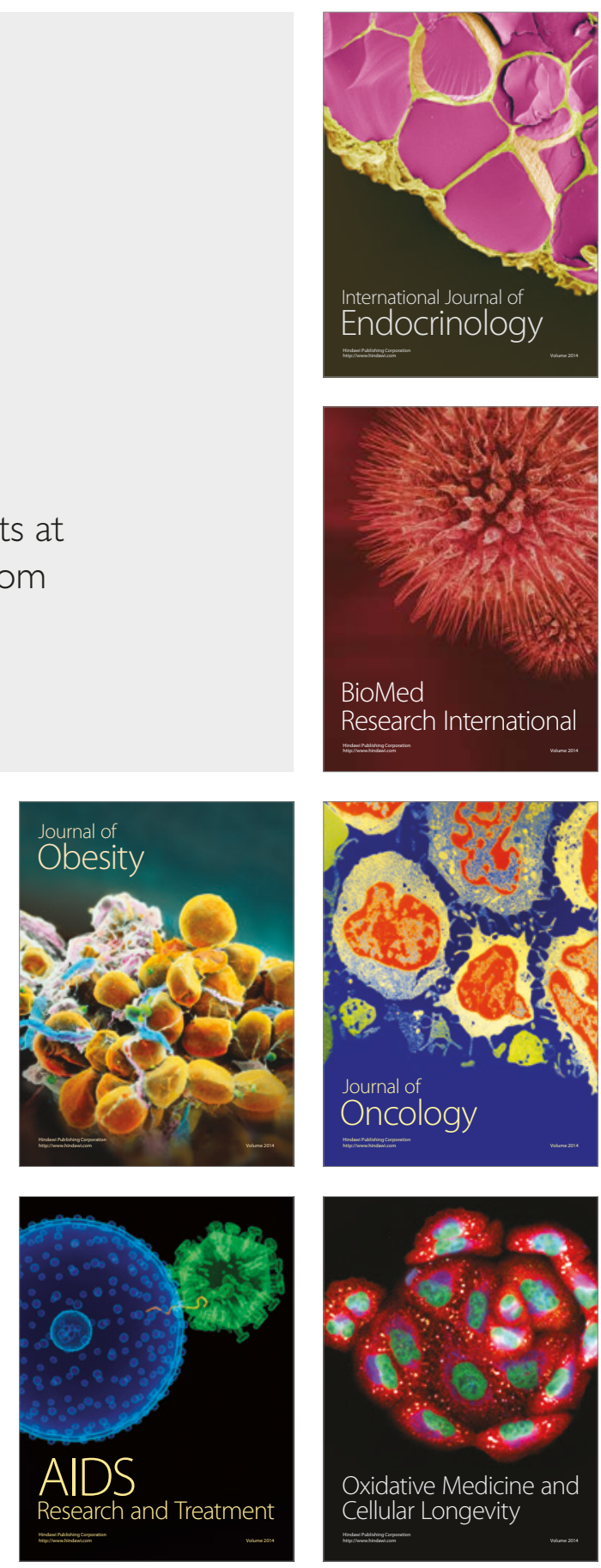